\title{
A Case of Malignant Small Round Cell Tumor of Temporal Bone with Facial Paralysis
}

\author{
Jae-Hong Lee, Jae-Hyun Seo, Kyung-Ho Park and Beom Cho Jun \\ Department of Otolaryngology-Head and Neck Surgery, The Catholic University of Korea College of Medicine, Seoul, Korea
}

$\begin{array}{ll}\text { Received } & \text { October 1, 2012 } \\ \text { Revised } & \text { October 21, 2012 } \\ \text { Accepted } & \text { October 22, } 2012\end{array}$

Address for correspondence

Beom Cho Jun MD, PhD

Department of Otolaryngology-

Head and Neck Surgery,

The Catholic University of Korea

College of Medicine,

Uijeongbu St. Mary's Hospital,

271 Cheonbo-ro,

Uijeongbu 480-717, Korea

Tel +82-31-820-3657

Fax $+82-31-847-0038$

E-mail otojun@catholic.ac.kr
Malignant small round cell tumor is very rare, especially in the head and neck area. It is also difficult to make a differential diagnosis due to their undifferentiated or primitive character. Immunohistochemical staining and chromosomal study is useful to categorize these tumors. Since these are rare tumors, treatment protocols are is not well established. While combined treatments (surgery, chemotherapy, radiotherapy) is currently being applied, the tumor still has a poor prognosis. We present a rare case of a rapidly growing temporal bone malignant small round cell tumor which initially showed facial paralysis.

Korean J Audiol 2012;16:145-147

KEY WORDS: Malignant small round cell tumor - Temporal bone.

\section{Introduction}

Malignant small round cell tumor (MSRCT) of temporal bone is very rare, with rapid aggressive patterns. Differential diagnosis of small round cell tumors is particularly difficult due to their undifferentiated or primitive character. ${ }^{1)}$ Many tumors belong to the category of "small, round, blue cell tumor". Rhabdomycosarcoma, lymphoma, lymphoblastic leukemia, small cell carcinoma, neuroblastoma and Ewing's sarcoma all have a similar histologic appearance. ${ }^{2)}$

For exact categorization of these tumors, immunohistochemistry and chromosomal study is essential.

The authors will describe a case of MSRCT of the temporal bone which showed negative results in all immunohistochemical studies.

\section{Case Report}

A 5-year old boy visited outpatient clinics for left idiopathic acute facial paralysis (House-Brackmann grade IV). Pure tone audiometry showed normal healing level. Oral steroid medication was prescribed for 10 days as scheduled during admission. On imaging study, temporal magnetic resonance imaging showed a partial fluid signal at the left mastoid air cells and abnormal enhancement at the left facial nerve around the genu area (Fig. 1). He was diagnosed with Bell's palsy. However there was no improvement until a month after discharge. His parents wanted to transfer to another hospital and returned six months later. He showed marked improvement without any treatment and there were no sequelae (HouseBrackmann grade I). Three months later, he visited again for left intractable otalgia without facial palsy. He had a medication history of local clinics for the last month. On physical examination, the left tympanic membrane could not be observed due to severe narrowing of left external auditory canal (Fig. 2). Pure tone audiometry showed sensorineuronal hearing loss of $60 \mathrm{~dB}$ threshold. Temporal bone computed tomography revealed expansile soft tissue densities and diffuse erosive destruction on left temporal bone (Fig. 3). On the basis of these findings, we suggested aggressive infectious disease or destructive cholesteatoma.

We performed an emergency modified radical mastoidectomy under general anesthesia. During the operation, we found a pale, grayish tumor occupying the mastoid and middle ear cavity (Fig. 4). On frozen biopsy, it was suspected to be a MSRCT 

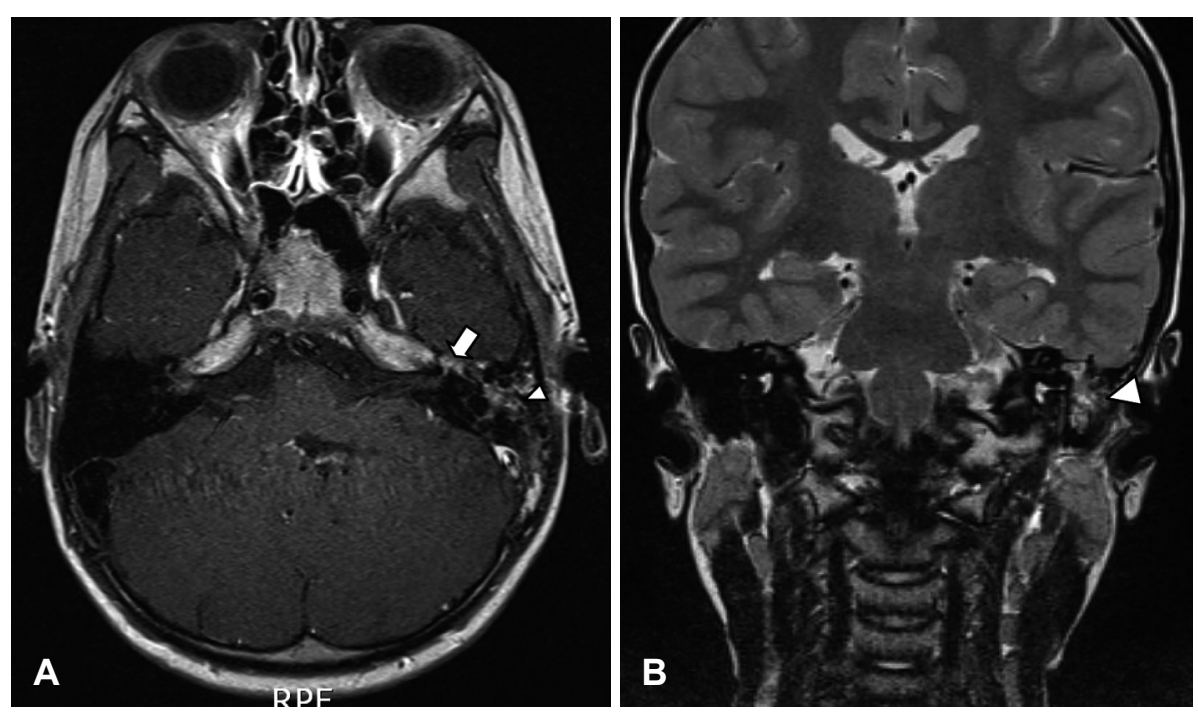

Fig. 1. Temporal bone magnetic resonance image. This shows partial fluid signal at left mastoid air cells (arrow head) and abnormal enhancement at Lt. facial nerve around genu area (arrow). A: Contrast T1 axial view. $\mathrm{B}$ : T2 coronal view.

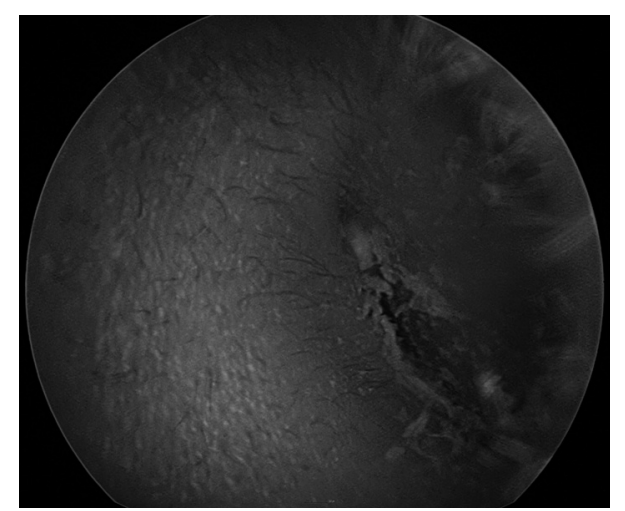

Fig. 2. Severely narrowed external auditory canal, left.

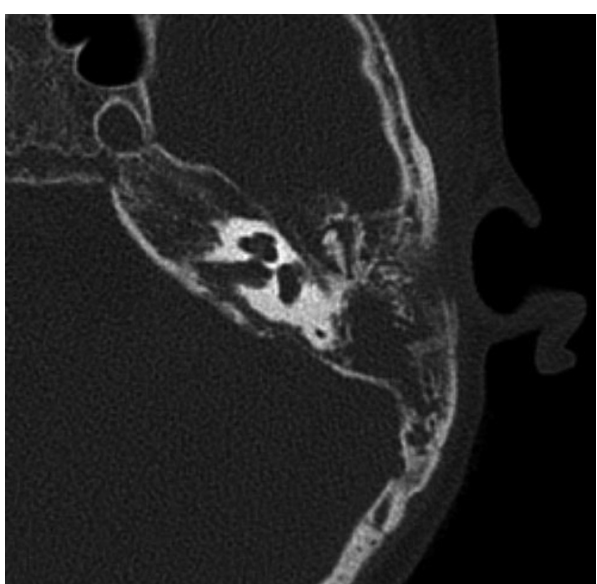

Fig. 3. Temporal bone computed tomography. This shows expansile soft tissue densities and diffuse erosive destruction on the left temporal bone.

Pathologic examination of the resected tumor showed an infiltrating tumor consisting of small round cells (Fig. 5). The results of immunohistochemical stainings for vimentin, neuron specific enolase (NSE), S-100 protein, pancytokeratin, synaptophysin, chromogranin A, smooth muscle actin, Sarcomer-

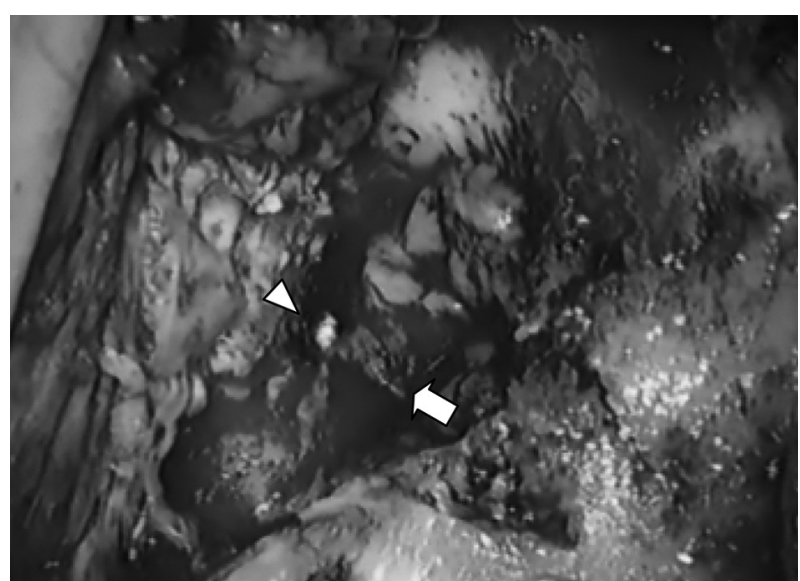

Fig. 4. Photomicrograph of left temporal bone tumor taken intraoperatively. There is a pale, grayish occupying mass extending to the mastoid (arrow) and middle ear cavity. Arrow head indicates the tympanic membrane.

ic actin, glial fibrillary acidic protein (GFAP), leuko-cyte common antigen were all negative, so we couldn't categorize the tumor.

After the pathologic confirmation, the patient transferred to hematooncologic department for chemotherapy and radiotherapy.

\section{Discussion}

Temporal bone and facial nerve involvement by a malignant tumor is not common; it comprises about $5 \%$ of peripheral paralysis causes, and malignant tumors are even rare. ${ }^{3)}$

MSRCT is a term used for tumors composed of malignant round cells that are slightly larger up to double the size of red blood cells in air-dried smears. They generally include Ewing's sarcoma, primitive neuroectodermal tumor (PNET), rhabdomyosarcoma, synovial sarcoma, non-Hodgkin's lympho- 


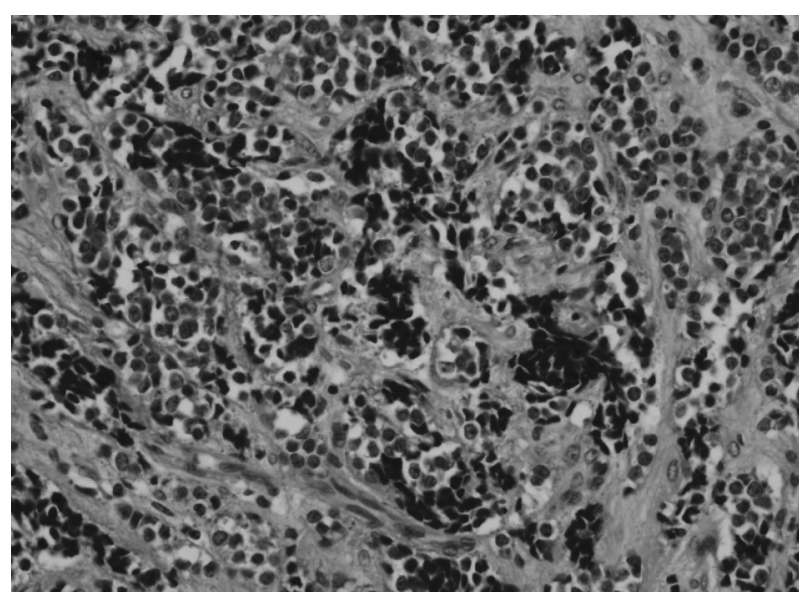

Fig. 5. Microscopically, H\&E staining shows small round cells with hyperchromatic nuclei and pale cytoplasm $(\times 400)$.

ma, retinoblastoma, neuroblastoma, hepatoblastoma and nephroblastoma. Differential diagnosis of small round cell tumors is particularly difficult due to their undifferentiated or primitive character. ${ }^{1)}$

For exact categorization of these tumors, immunohistochemistry and chromosomal study is useful. Immunohistochemistry is crucial in distinguishing Ewing's sarcoma from PNET. Recently NSE, S-100 protein, GFAP, synaptophysin, neural cell adhesion molecule and protein gene product 9.5 have been clinically tested as neural antibodies for establishing a diagnosis for PNET. ${ }^{4)}$ In chromosomal study, Ewing sarcoma/ PNET typically shows a recurrent chromosomal translocation, most commonly involving chromosomes 11 and 22 resulting in the fusion of the genes EWS and FLI. ${ }^{5)}$ In the present case, staining with all markers was negative.

The occurrence of these tumors in head and neck is very rare. Tiago, et al. ${ }^{6}$ reported a case of PNET of temporal bone. Yamazaki, et al. ${ }^{4)}$ reported a case of PNET of jugular foramen.
Jones and $\mathrm{McGill}^{7)}$ reported 11 cases in the head and neck region out of 26 patients with PNET.

The treatment protocol of MSRCT is not yet been established. Combined treatment (surgery, chemotherapy and radiotherapy) is currently applied depending on the tumor size and location although the prognosis remains poor. Eralp, et al. ${ }^{8)}$ reported a 30.5\% 3-year disease free and overall survival rate.

We presented a rare case of MSRCT of temporal bone. MSRCT showed an aggressive clinical pattern. Multicenter and large scale treatment outcomes and treatment protocol are required.

\section{REFERENCES}

1) Rajwanshi A, Srinivas R, Upasana G. Malignant small round cell tumors. J Cytol 2009;26:1-10.

2) Nikitakis NG, Salama AR, O’Malley BW Jr, Ord RA, Papadimitriou JC. Malignant peripheral primitive neuroectodermal tumor-peripheral neuroepithelioma of the head and neck: a clinicopathologic study of five cases and review of the literature. Head Neck 2003;25:488-98.

3) Boahene DO, Olsen KD, Driscoll C, Lewis JE, McDonald TJ. Facial nerve paralysis secondary to occult malignant neoplasms. Otolaryngol Head Neck Surg 2004;130:459-65.

4) Yamazaki T, Kuroki T, Katsume M, Kameda N. Peripheral primitive neuroectodermal tumor of the jugular foramen: case report. Neurosurgery 2002;51:1286-9; discussion 1289

5) Salgado C, Neff T, Frazier L, Vargas SO, Vanderveen D. An unusual case of congenital primitive neuroectodermal tumor with ocular metastasis. J Pediatr Hematol Oncol 2012;34:e69-71.

6) Tiago RS, Pio MR, Silva MN, do Valle LO. Peripheral primitive neuroectodermal tumor: a rare case of peripheral facial paralysis. Braz J Otorhinolaryngol 2007;73:136.

7) Jones JE, McGill T. Peripheral primitive neuroectodermal tumors of the head and neck. Arch Otolaryngol Head Neck Surg 1995;121: 1392-5.

8) Eralp Y, Bavbek S, Başaran M, Kaytan E, Yaman F, Bilgiç B, et al. Prognostic factors and survival in late adolescent and adult patients with small round cell tumors. Am J Clin Oncol 2002;25:418-24. 